\title{
openheart MiR-223-3p and miR-122-5p as circulating biomarkers for plaque instability
}

\author{
Sandeep Singh (10 , ${ }^{1,2}$ Maurice W J de Ronde, ${ }^{1,2}$ Maayke G M Kok, ${ }^{1}$ \\ Marcel AM Beijk, ${ }^{3}$ Robbert J De Winter, ${ }^{3}$ Allard C van der Wal, ${ }^{4}$ \\ Brigitte M Sondermeijer, ${ }^{1}$ Joost C M Meijers, ${ }^{5,6}$ Esther E Creemers (D) , ${ }^{7}$ \\ Sara-Joan Pinto-Sietsma ${ }^{1,2}$
}

\begin{abstract}
- Additional material is published online only. To view, please visit the journal online (http://dx.doi.org/10.1136/ openhrt-2019-001223).
\end{abstract}

To cite: Singh S, de Ronde MWJ, Kok MGM, et al. MiR-223-3p and miR-122-5p as circulating biomarkers for plaque instability. Open Heart 2020;7:e01223. doi:10.1136/ openhrt-2019-001223

SS and MWJdR contributed equally.

SS and MWJdR are joint first authors.

Received 10 December 2019 Revised 31 March 2020 Accepted 10 April 2020
Check for updates

(C) Author(s) (or their employer(s)) 2020. Re-use permitted under CC BY-NC. No commercial re-use. See rights and permissions. Published by BMJ.

For numbered affiliations see end of article.

Correspondence to Dr Sara-Joan Pinto-Sietsma; pintosj@gmail.com

\section{ABSTRACT}

Background In this study, we discovered and validated candidate microRNA (miRNA) biomarkers for coronary artery disease (CAD).

Method Candidate tissue-derived miRNAs from atherosclerotic plaque material in patients with stable coronary artery disease (SCAD) $(n=14)$ and unstable coronary artery disease (UCAD) $(n=25)$ were discovered by qPCR-based arrays. We validated differentially expressed miRNAs, along with seven promising CAD-associated miRNAs from the literature, in the serum of two large cohorts ( $n=395$ and $n=1000$ ) of patients with SCAD and UCAD and subclinical atherosclerosis (SubA) and controls, respectively.

Result From plaque materials (discovery phase), miR$125 b-5 p$ and miR-193b-3p were most upregulated in SCAD, whereas miR-223-3p and miR-142-3p were most upregulated in patients with UCAD. Subsequent validation in serum from patients with UCAD, SCAD, SubA and controls demonstrated significant upregulation of miR-223-3p, miR-133a-3p, miR-146-3p and miR-155$5 p$. The ischaemia-related miR-499-5p was also highly upregulated in patients with UCAD compared with the other groups (SCAD OR 20.63 (95\% Cl 11.16 to 38.15), SubA OR 96.10 (95\% Cl 40.13 to 230.14) and controls OR 15.73 (95\% Cl 7.80 to 31.72)). However, no significant difference in miR-499-5p expression was observed across SCAD, SubA and controls. MiR-122-5p was the only miRNA to be significantly upregulated in the serum of both patients with UCAD and SCAD.

Conclusion In conclusion, miR-122-5p and miR-223-3p might be markers of plaque instability.

\section{INTRODUCTION}

Cardiovascular disease is one of the leading causes of death worldwide. ${ }^{1}$ However, early detection of coronary artery disease (CAD) remains a challenge. Therefore, biomarkers are needed to improve prognostication and to individualise treatment. Nowadays, the risk of CAD is assessed by algorithms based on patients' risk factors such as the American College of Cardiology/American Heart Association or Systematic COronary Risk

\section{Key questions}

What is already known about this subject?

- Several circulating microRNAs (miRNAs) have been reported to differentially express between healthy individuals and patients with coronary artery disease (CAD).

- Unfortunately, none of the identified circulating miRNA biomarkers was strong enough to make it to clinical practice.

What does this study add?

- In this large cross-sectional study, we were able to show that tissue-derived miR-223-3p and serum miR-122-5p are robust and promising biomarkers for unstable $\mathrm{CAD}$ disease, possibly reflecting plaque instability.

- Besides, miR-122-5p could also reflect an adverse metabolic profile increases in the risk for atherosclerosis.

How might this impact on clinical practice?

- Existing CAD risk algorithms do not take into the account that CAD can either remain stable (SCAD) or can become unstable (UCAD).

- Therefore, these miRNAs could be helpful in risk prediction for (early) CAD as well as markers that can discriminate SCAD from UCAD.

Evaluation (SCORE) calculators. ${ }^{2} 3$ Unfortunately, these risk score algorithms provide a poor estimate of an individual's risk, especially in young individuals. Besides, these algorithms do not take into account that CAD can either remain stable (SCAD) or can become unstable (UCAD). Although atherosclerosis is their common factor, SCAD and UCAD have completely different pathophysiology and outcome. ${ }^{4}$ SCAD can remain stable for many years and may cause cardiac complaints during exercise, yet it does not immediately jeopardise the myocardium. Whereas, in patients with $\mathrm{UCAD}$, the atherosclerotic plaque may erode or even rupture and as such cause an acute 
coronary syndrome, which can lead to loss of myocardium by ischaemia and eventually heart failure and/or death. ${ }^{5}$ Therefore, for accurate cardiovascular risk prediction and early treatment, it is crucial to have markers that can discriminate SCAD from UCAD in an early phase.

miRNAs are short, non-coding RNAs that posttranscriptionally regulate gene expression ${ }^{6}$ by binding the $3^{\prime}$ untranslated region of target gene mRNAs. ${ }^{7}$ miRNAs are noted as key players in modulating the function of endothelial cells, smooth muscle cells and macrophages, regulating atherosclerosis pathogenesis. ${ }^{8-11}$ They are ideal biomarkers since they are quite tissue-specific and remain stable in the blood, ${ }^{12} 13$ but an optimal performing biomarker should only present in the circulation in the diseased state and absent in healthy controls. This poses a problem for CAD since one would ideally need biopsy material to be able to investigate the most abundant miRNAs for CAD. Nowadays, there are a few studies on miRNA expression profiles in atherosclerotic plaques, ${ }^{81415}$ but because of the often small sample sizes, these studies are prone to bias, because of small sample errors and issues with reproducibility (ref: small sample size paper).

In this study, we aimed to overcome the issues of small sample error and reproducibility, by showing robust outcomes of miRNAs for either SCAD or UCAD from discovery to multiple validations. Explicitly this study was not intended to explore the mechanisms of the proposed miRNAs, but to explore the reproducibility of earlier discovered atherosclerotic plaque miRNAs, as a robust marker of SCAD or UCAD. We used an epidemiological approach using larger sample sizes and multiple validation methods, to overcome small sample errors. Therefore, this study, on miRNA biomarkers for SCAD and UCAD, comprises a discovery phase, a first validation of the discovery phase and a second validation to be able to further substantiate the first validation.

\section{METHODS}

A flow chart of the distinct cohorts and their properties used in the discovery and validation phase of the study is presented in figure 1.

\section{Source populations}

For our study, we made use of two different source populations A and B. In short, source population A consisted of patients who underwent a percutaneous coronary intervention, electively or because of acute coronary syndrome. Source population B consisted of serum samples from a biobank of patients with premature atherosclerosis and their 'healthy' family members. From these two source populations, we selected three study populations (a, b and c). For details on source and study populations, see online supplementary methods.

\section{Study populations}

\section{Discovery cohort}

The discovery cohort a $(n=39)$ consisted of patients with an acute coronary syndrome (UCAD group; $n=25$; Aa) or stable angina ( $\mathrm{SCAD}$ group; $\mathrm{n}=14 ; \mathrm{Aa}$ ) from whom tissue of either thrombectomy or atherectomy material from coronary angiography was available. Further detailed information on tissue collection is described in online supplementary methods.

\section{First validation cohort}

The first validation cohort $b(n=395)$ consisted of patients with an acute coronary syndrome (UCAD group; $n=64$; $\mathrm{Ab}$ ), or stable angina (SCAD group; $\mathrm{n}=139 ; \mathrm{Ab}$ ) and controls (control; $\mathrm{n}=192 ; \mathrm{Bb}$ ). Controls did not have any detectable atherosclerosis as indicated by an Agatston coronary artery calcium (CAC) score of 0 determined by coronary CT scan.

\section{Second validation cohort}

The second validation cohort $\mathrm{c}(\mathrm{n}=1000)$ consisted of patients with an acute coronary syndrome (UCAD group; $\mathrm{n}=250$; Ac), or patients with a history of CAD, without cardiac complains (SCAD group; $n=250 ; \mathrm{Bc}$ ), or patients with subclinical atherosclerosis, as indicated by a CAC score of $\geq 1$, without cardiac complains (SubA group; $\mathrm{n}=250 ; \mathrm{Bc}$ ) and controls with a CAC score of 0 (control; $\mathrm{n}=250 ; \mathrm{Bc}$ ).

\section{Blood withdrawal}

Serum samples were drawn before the administration of heparin and were stored in cryovials at $-80^{\circ} \mathrm{C}$ as described in online supplementary methods.

\section{Selection of candidates for the first validation phase}

Tissue-derived miRNAs from the discovery cohort were ranked, as described in online supplementary methods, for the first validation candidate selection.

\section{Selection of miRNAs for the second validation phase}

To be able to show the robustness of our results, we decided to revalidate these results in a second validation phase. The first validation phase showed that miR125b-5p, miR-223-3 p, miR-142-3 p and miR-193b-3p could be promising biomarkers for either stable or unstable cardiovascular disease. These miRNA together with the most promising miRNA biomarkers for cardiovascular disease published, namely miR-133a-3p, miR-146-3p, miR-126-3 p, miR-145-5 p, miR-155-5 p and miR-122-5 p, were measured in the second validation phase. For the literature miRNAs, we conducted a literature search and selected all miRNAs that were consistently upregulated or downregulated in more than two studies. ${ }^{916-19}$ To be able to differentiate whether the candidate miRNAs were due to ischaemia, we also added an ischaemia-related miR-499-5 p to our panel. ${ }^{20}$

\section{RNA isolation and quantification of miRNAs by RT-qPCR}

Procedure on RNA isolation, complementary DNA synthesis, quantification, RT-qPCR data handling, and normalisation are described in online supplementary methods. 


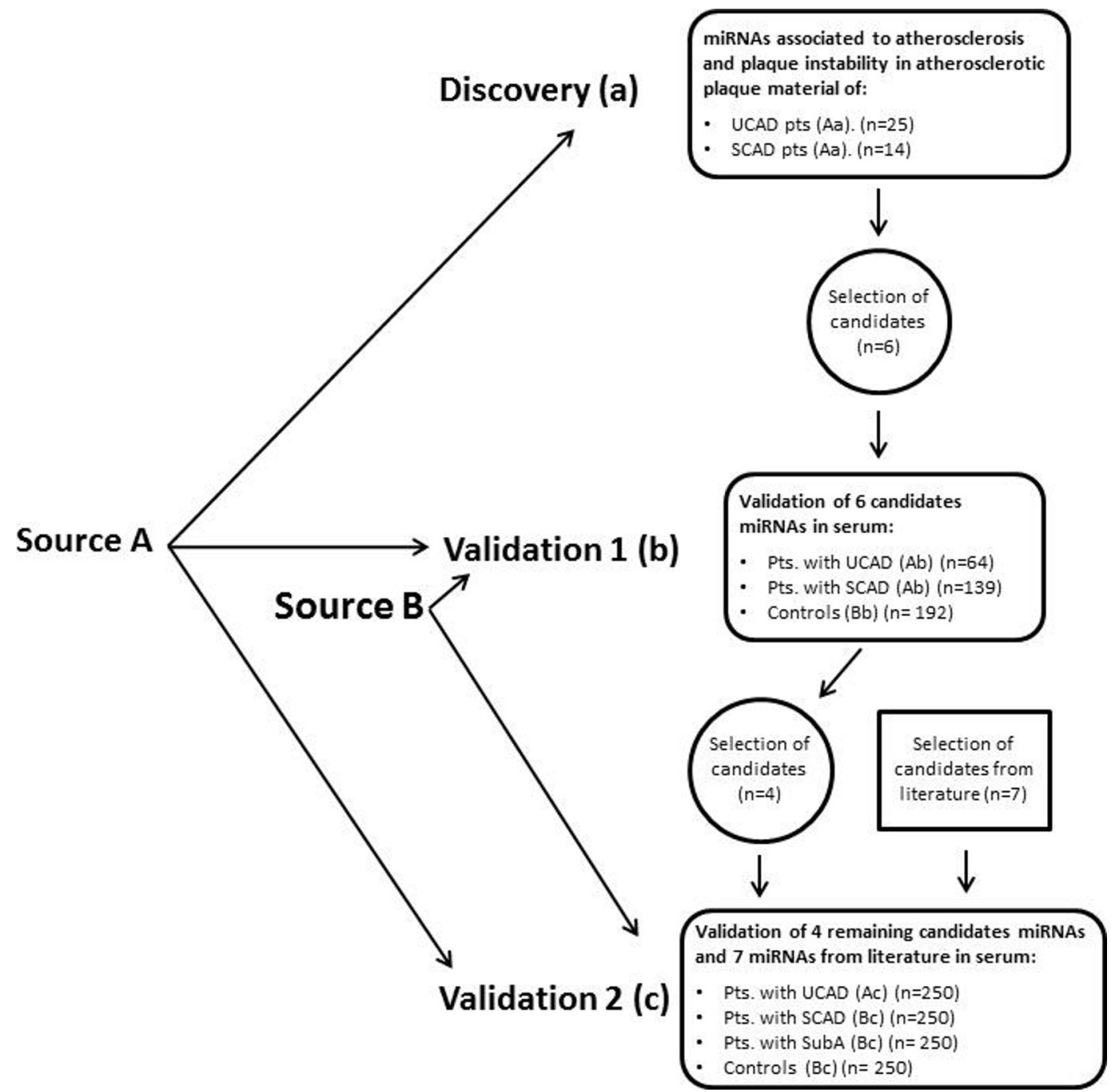

Figure 1 Flow chart of the study outline. miRNA, microRNA; SCAD, stable coronary artery disease; SubA, subclinical atherosclerosis; UCAD, unstable coronary artery disease.

Biochemical parameters

Liver enzymes and lipids were measured as described in the online supplementary methods.

\section{Coronary CT scan}

Coronary artery CT scan was performed as described in the online supplementary methods.

\section{Patient and public involvement}

It was not appropriate or possible to involve patients or the public in the design, conduct, reporting, or dissemination plans of our research.

\section{Statistical analysis}

Baseline characteristics are expressed as mean $\pm \mathrm{SD}$ for continuous variables and number (\%) for dichotomous variables, except when indicated otherwise. Analysis of variance with post hoc Student's t-test, Mann-Whitney U test and Fisher's exact test was used to calculate differences in baseline characteristics as appropriate. In the discovery phase, T-tests were used to calculate differences in array expression which were expressed in a mean difference of normalised $\mathrm{C}_{\mathrm{q}}$ values. $\mathrm{P}$ values of the $\mathrm{qPCR}$-based array were Benjamini-Hochberg corrected for multiple testing. In the validation phase, logistic regression was used to analyse the differences in miRNA expression between the SCAD, UCAD and the control group expressed as normalised log-transformed starting concentrations $\left(\mathrm{N}_{0}\right)$ as calculated by LinRegPCR. ${ }^{21}$ In the validation cohort, multivariate analyses were performed on two different models to adjust for age and gender. All statistical analyses were performed using SPSS for Windows V.23. A p value $<0.05$ was considered to be statistically significant.

\section{RESULTS}

\section{Discovery phase}

qPCR-based miRNA array on tissue samples

The clinical characteristics of the discovery cohort are shown in table 1 . Of the 742 measurable tissue-derived miRNAs, 125 qualified for reliable analysis as described in the 'Methods' section and were included in the analysis. In total, 54 tissue-derived miRNAs were significantly 


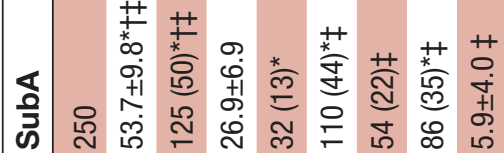

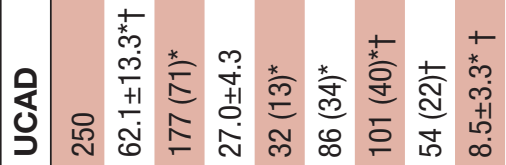

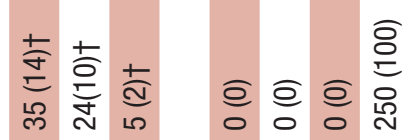

응

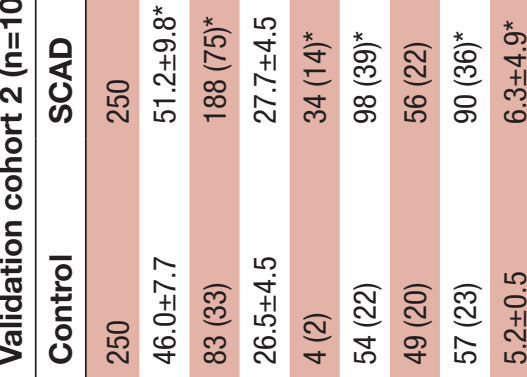

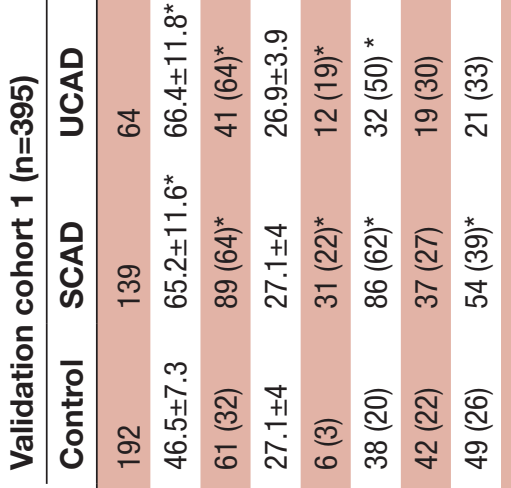

\section{จุ}

II

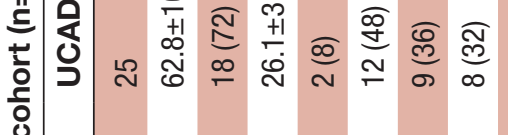

\section{ग)}

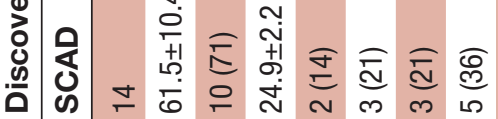

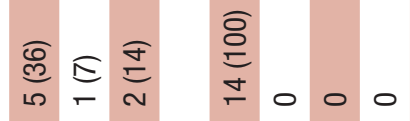

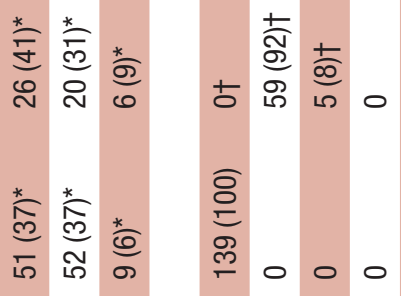

000

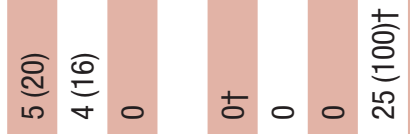

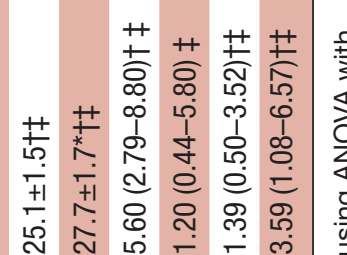

茛吉 $\frac{+}{\frac{*}{2}} \frac{+}{*}$

t *

$+1+1$ 立

సं

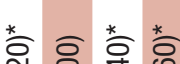

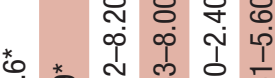

*

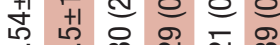



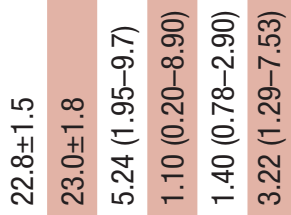

ఖ্ষ

a

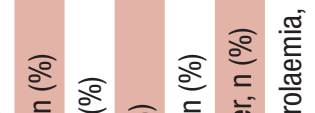

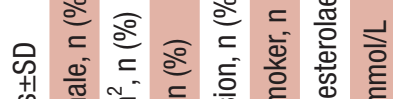

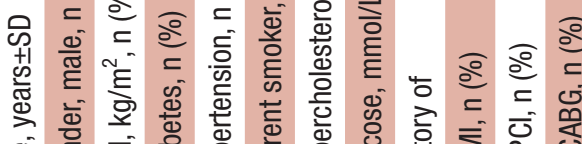

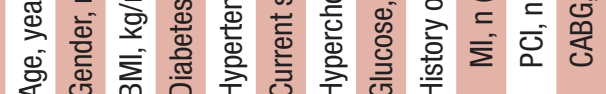

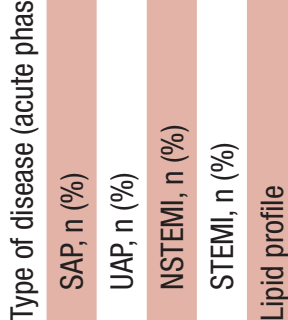

흘

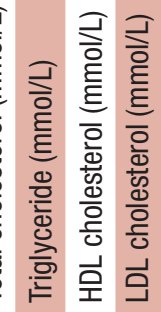

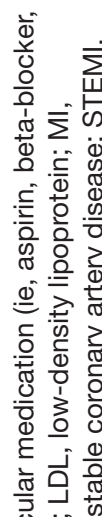

容

응 엉

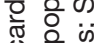

을 흉

응

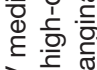

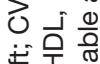

武定需

केष

需

승

>

(1)

ㅎํ윰

ह $\frac{\mathcal{C}}{\mathrm{C}}$

한

흥

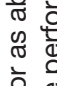

흐

娄

흥

을

흥

들

要

का 응

零.

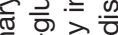

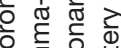

论 능

पॅ

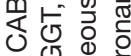

齐要

.

की

हैं ठ்

ते



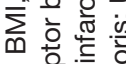

बi ه

का

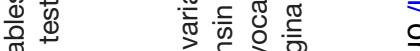

त

옹 음 등



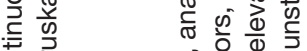

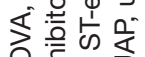

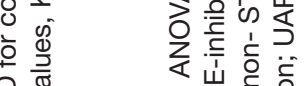

की

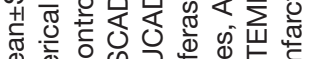

\%

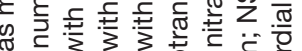

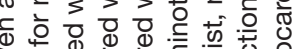

क

का

ธै

क)

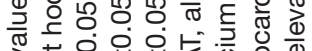




upregulated in SCAD compared with UCAD and 50 tissue-derived miRNAs were significantly upregulated in UCAD compared with SCAD. For expression levels of all tissue-derived miRNAs, see online supplementary table 1.

The top three most upregulated tissue-derived miRNAs in SCAD were miR-125b-5p (mean diff: 5.94), miR-455-3p (mean diff: 5.66) and miR-193b-3p (mean diff: 5.66) and in UCAD were miR-223-3 $p$ (mean diff: 2.55), miR-142-3p (mean diff: 2.24) and miR-126-5 p (mean diff: 1.77).

Of these tissue-derived miRNAs, miR-455-3p and miR-126-5p could not be measured in serum. For miR-455-3p, the expression level was undetectable and for miR-126-5 $p$ the cDNA synthesis failed and there was not enough material to repeat the analyses. Therefore, four out of the six candidate tissue-derived miRNAs were validated: miR-125b-5p, miR-193b-3p, miR-223-3p and miR-142-3 p.

\section{First validation}

Multivariate analysis of the four candidate tissue-derived miRNAs in serum

Clinical characteristics of validation cohort 1 are shown in table 1 . We performed a multivariate analysis correcting for age and gender, in the first validation. We were able to validate, in serum, all four candidate tissue-derived miRNAs observed in our discovery experiment against controls. We were able to show that miR-125b-5p (OR 2.09, $95 \%$ CI 1.30 to 3.36), miR-193b-3p (OR 1.41, 95\% CI 1.00 to 2.00 ), miR-223-3p (OR 1.61, 95\% CI 1.20 to 2.15 ) and miR-142-3p (OR 1.38, 95\% CI 1.05 to 1.82 ) (figures 2 and 3 and online supplementary table 3 ) were significantly upregulated in patients with SCAD as compared with controls. On the other hand, we were only able to validate the difference in SCAD versus UCAD for miR125b-5p (OR 1.64, 95\% CI 1.09 to 2.48). On the other hand, there was a slight non-significant higher expression of miR-193b-3p in SCAD as compared with UCAD, similar as observed in the discovery experiment. In addition, for patients with UCAD miR-223-3p (OR 2.38, 95\% CI 1.41 to 4.01 ) and miR-142-3p (OR 1.62, 95\% CI 1.12 to 2.35 ) also showed a significant difference with controls. Combined, these results suggest that all four tissue-derived miRNAs could be validated in serum and might serve as markers for the presence of coronary atherosclerosis and to a lesser extent, miR-223-3 $p$ might serve as a marker for UCAD.
Discovery

A

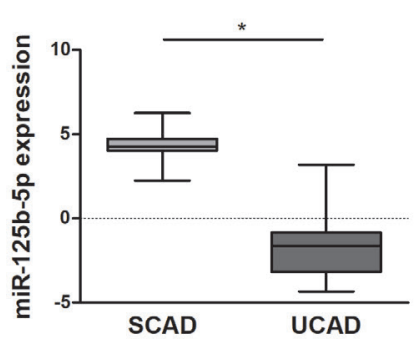

D

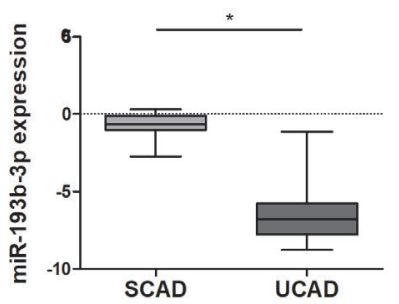

Validation 1

B

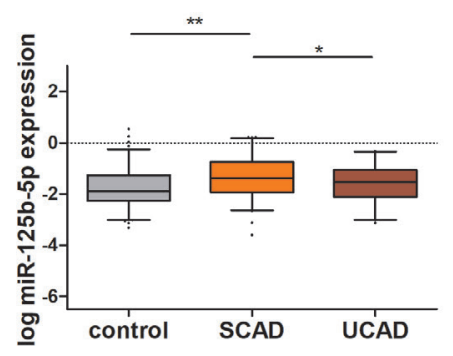

E

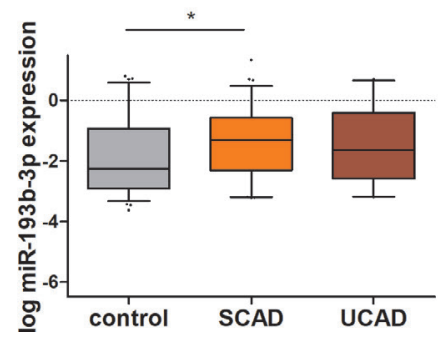

Validation 2

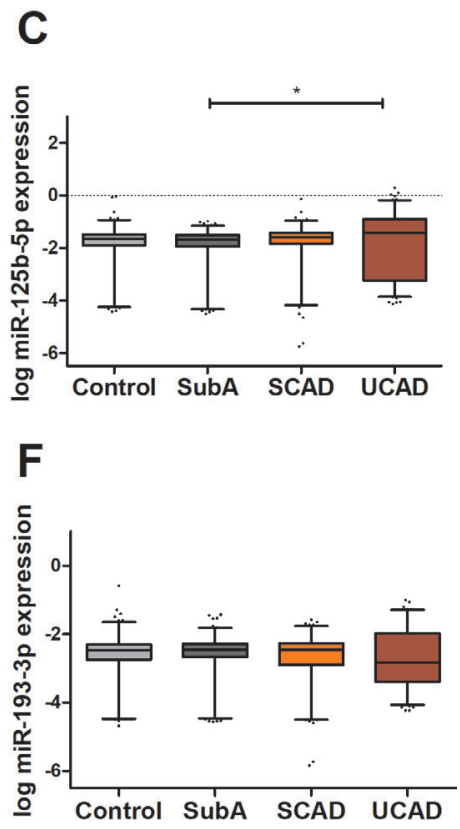

Figure 2 Expression levels of microRNAs (miRNAs) upregulated in SCAD (miR-125b-5p and miR-193b-3p). Normalised expression levels are shown for both the discovery and the validation experiments. Expression levels of miR-125b-5p (A) and miR-193b-3p (D) in both atherectomy (SCAD, $n=14$ ) and thrombectomy material (UCAD, $n=25)$ were determined by qPCRbased array in the discovery experiments. In the first validation phase, log-transformed expression levels of miR-125b-5p (B) and miR-193b-3p (E) were determined by serum qPCR measurement in patients with UCAD ( $n=64)$, SCAD ( $n=139)$ and controls $(n=192)$. In the second validation phase, log-transformed expression levels of miR-125b-5p (C) and miR-193b-3p (F) were determined by serum qPCR in patients with UCAD $(n=250)$, SCAD $(n=250)$, SubA $(n=250)$ and controls $(n=250)$. Statistical analyses in the validation phases were corrected for age and gender. More detailed information on expression level differences is found in online supplementary table 3 (first validation) and online supplementary table 5 (second validation). SCAD, stable coronary artery disease, SubA, subclinical atherosclerosis (ie, coronary calcium score $\geq 1$ on coronary CT scan); UCAD, unstable coronary artery disease. ${ }^{*} \mathrm{P}<0.05,{ }^{* *} \mathrm{p}<0.01$. 
Discovery

A

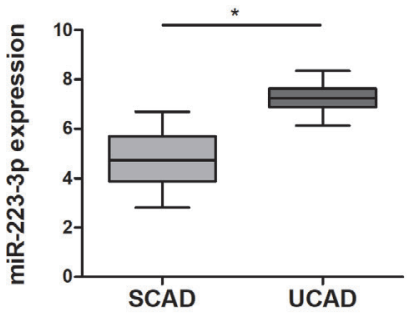

D

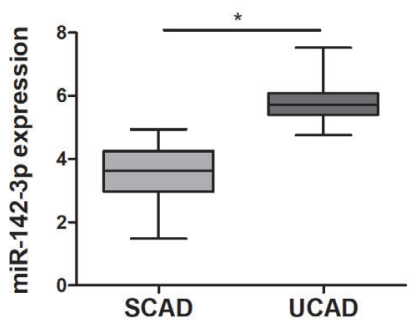

Validation 1

B

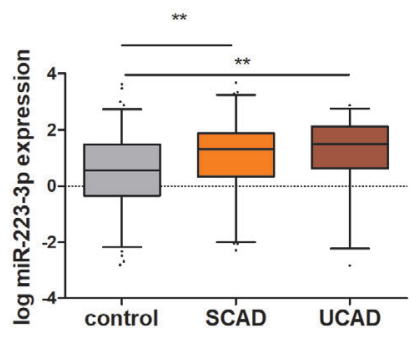

E

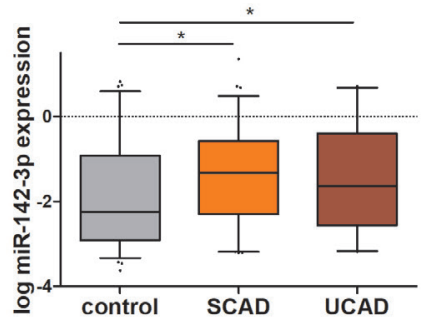

Validation 2

C

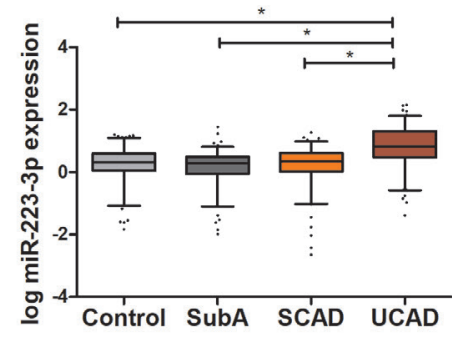

$\mathbf{F}$

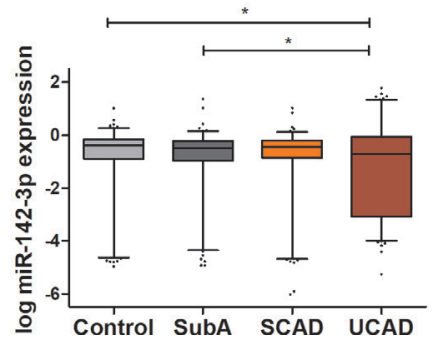

Figure 3 Expression levels of microRNAs (miRNAs) upregulated in UCAD (miR-223-3p, miR-142-3 p). Normalised expression levels are shown for both the discovery and the validation experiments. Expression levels of miR-223-3p (A) and miR-142-3 $p$ (D) in both atherectomy (SCAD, $n=14$ ) and thrombectomy material (UCAD, $n=25)$ were determined by qPCR-based array in the discovery experiments. In the first validation phase, log-transformed expression levels of miR-223-3p (B) and miR-142-3 $p$ (E) were determined by serum qPCR measurement in patients with UCAD ( $n=64)$, SCAD $(n=139)$ and controls $(n=192)$. In the second validation phase, log-transformed expression levels of miR-223-3 $p(C)$ and miR-142-3p (F) were determined by serum qPCR in patients with UCAD $(n=250)$, SCAD $(n=250)$, SubA $(n=250)$ and controls $(n=250)$. Statistical analyses in the validation phases were corrected for age and gender. More detailed information on expression level differences is found in online supplementary table 3 (first validation) and online supplementary table 5 (second validation). SCAD, stable coronary artery disease; SubA, subclinical atherosclerosis (ie, coronary calcium score $\geq 1$ on coronary CT scan); UCAD, unstable coronary artery disease. ${ }^{*} \mathrm{P}<0.05,{ }^{* *} \mathrm{p}<0.01$.

\section{Second validation}

The previous data imply that the discovered tissue-derived miRNA markers can also detect the presence of atherosclerosis in the serum of these patients. Since circulating miRNA biomarkers for the disease are often not being replicated in other studies, we deemed it necessary to try and replicate this finding ourselves in a second, larger cohort. Besides, for replication purposes, we added miRNAs from the literature which were suggested to be associated with CAD.

\section{Multivariate analysis of the candidate miRNAs from the second} validation study

The clinical characteristics of the validation cohort are shown in table 1 . In our second validation study, in which we did a multivariate analysis correcting for age and gender, we were able to validate, in serum, one out of the four candidate tissue-derived miRNAs identified from our discovery experiment. MiR-223-3 p was significantly upregulated in the UCAD group compared with controls (OR 13.92 (95\% CI 7.29 to 26.57) and patients with SCAD (10.75 (95\% CI 6.46 to 17.89); figure 3 and online supplementary table 5). This miRNA was also significantly upregulated when compared with patients with SubA (OR 12.75 (95\% CI 7.42 to 21.91). Concerning the other candidate miRNAs from the discovery experiment, miR-142-3 p showed an opposite effect, namely a significant downregulation as compared with controls (OR 0.90 (95\% CI 0.75 to 1.07)) and patients with SubA (OR 0.83 (95\% CI 0.72 to 0.96$)$ ) when compared with patients with UCAD.

Multivariate analysis of the candidate miRNAs from literature

Age-corrected and gender-corrected multivariate analysis of the CAD-related miRNAs from the literature (figure 4, online supplementary table 5) showed an elevated expression, in serum, in patients with UCAD of miR-122-5 p, miR-146-3 $p$ and miR-155-5p with all other subgroups (OR and $95 \% \mathrm{CI}$, online supplementary table 5). On the other hand, miR-145-5 p was significantly downregulated, in serum, in patients with UCAD as compared with the other subgroups. Furthermore, miR-122-5p was significantly upregulated in patients with UCAD versus the other groups, and patients with SCAD as compared with both SubA OR 1.38 (95\% CI 1.07 to 1.77$)$ and controls OR 1.45 (95\% CI 1.09 to 1.93). The ischaemia-related miR-499-5 p was also highly upregulated in patients with UCAD compared with the other patient groups (SCAD 


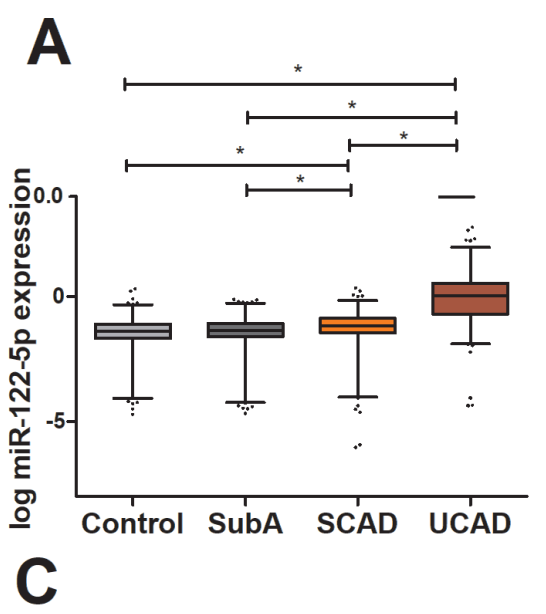

\section{B}
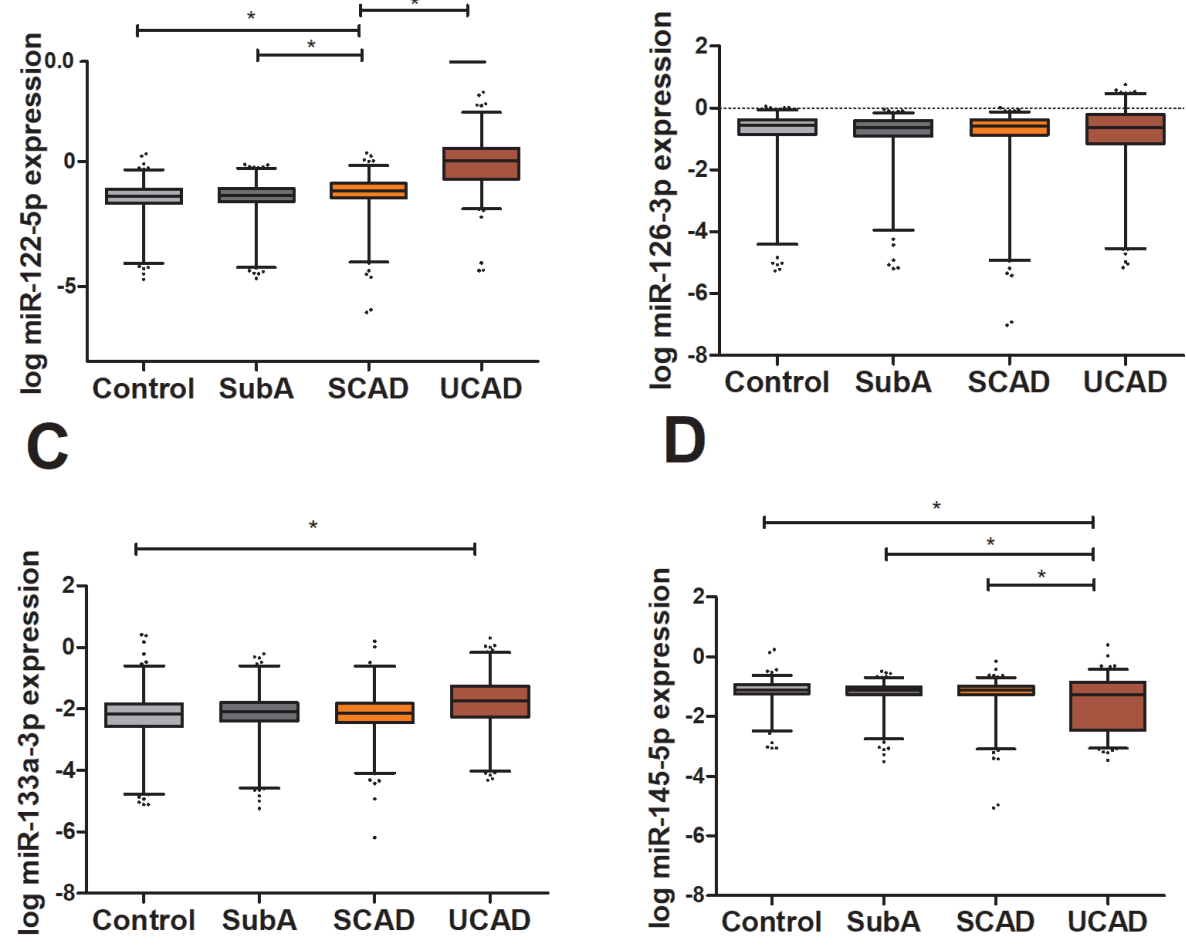

$\mathbf{E}$

$\mathbf{F}$
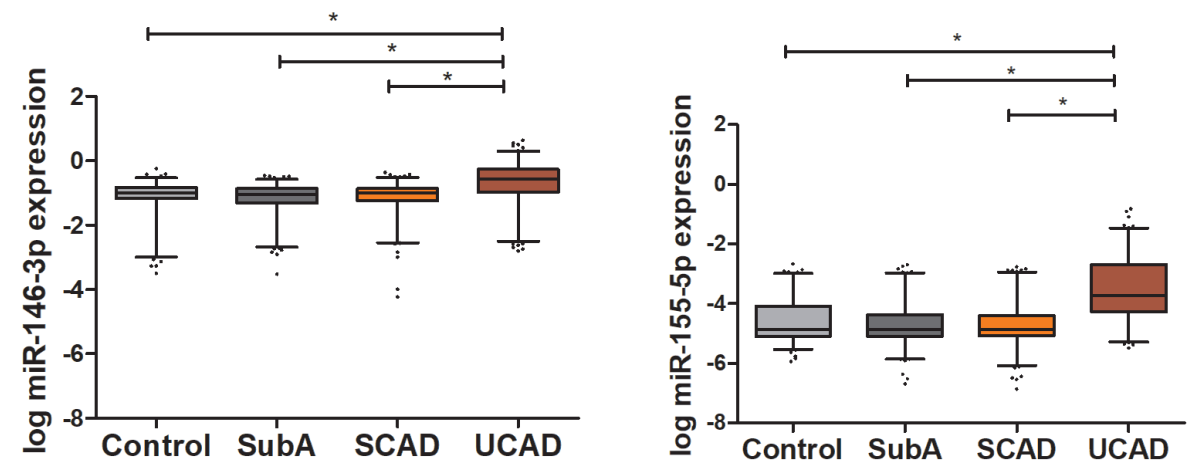

G

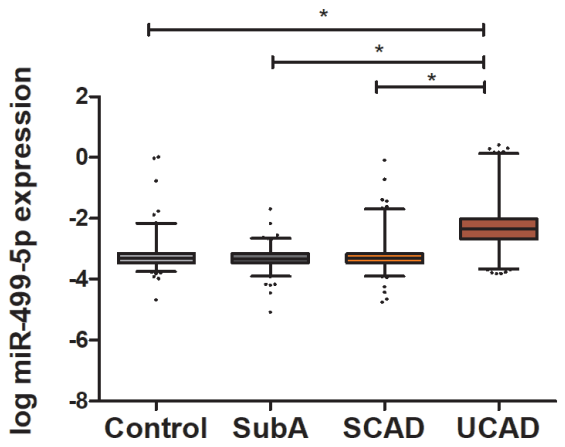

Figure 4 MicroRNA (miRNA) expression levels of atherosclerosis-related miRNAs from literature. Graphs show log transformed and normalised concentrations of miR-122-5p (A), miR126-3p (B), miR133a-3p (C), miR145-5p (D), miR-146-3p (E), miR-155-5p (F) and miR-499-5p (G). SCAD, stable coronary artery disease; SubA, subclinical atherosclerosis (ie, coronary calcium score $>0$ on coronary CT scan); UCAD, unstable coronary artery disease. ${ }^{*} \mathrm{P}<0.05$. 


\begin{tabular}{|c|c|}
\hline Model & AUC \\
\hline \multicolumn{2}{|l|}{ Model I } \\
\hline Age and gender & 0.79 \\
\hline \multicolumn{2}{|l|}{ Model II } \\
\hline miR-122-5p & 0.63 \\
\hline \multicolumn{2}{|l|}{ Model III } \\
\hline miR-223-3p & 0.50 \\
\hline \multicolumn{2}{|l|}{ Model IV } \\
\hline Age and gender+miR-122-5p+miR-223-3p & 0.80 \\
\hline \multicolumn{2}{|c|}{ AUC for ROC curve for UCAD compared with controls } \\
\hline \multicolumn{2}{|l|}{ Model I } \\
\hline Age and gender & 0.88 \\
\hline \multicolumn{2}{|l|}{ Model II } \\
\hline miR-122-5p & 0.90 \\
\hline \multicolumn{2}{|l|}{ Model III } \\
\hline miR-223-3p & 0.76 \\
\hline \multicolumn{2}{|l|}{ Model IV } \\
\hline Age and gender+miR-122-5p+miR-223-3p & 0.96 \\
\hline
\end{tabular}

$A \cup C$, area under the curve; ROC, receiver operating characteristic.

OR 20.63 (95\% CI 11.16 to 38.15), SubA OR 96.10 (95\% CI 40.13 to 230.14)) and controls OR 15.73 (95\% CI 7.80 to 31.72 ) and might indicate that most of the miRNAs that are upregulated in the patients with UCAD reflect acute ischaemia. Indeed, this could be the case for all miRNAs upregulated in patients with UCAD, except for miR-122-5 p, which was also upregulated in patients with SCAD. If the upregulation of miR-122-5 $\mathrm{p}$ in patients with UCAD would be an indication for acute ischaemia, this would mean that patients with SCAD with an upregulated miR-122-5 $\mathrm{p}$ would have acute ischaemia, although they did not have any complaints. Since miR-499-5 p was only upregulated in UCAD and not in SCAD, this seems unlikely. Furthermore, in a linear regression model, no significant association was noticed between hsTnI and miR-122-5 p (data not shown).

Finally, we also performed a receiver operating characteristic analysis in which we were able to show that miR-122-5 $\mathrm{p}$ and miR-223-3 $\mathrm{p}$ when considered together in a model that identified SCAD and UCAD with an area under the curve of 0.80 and 0.96 , respectively (table 2).

To conclude, the second validation, miR-122-5p, miR-146-3p, miR-155-5p and miR-145-5p could be promising markers for unstable CAD. Besides, since miR-122-5 $p$ was also significantly upregulated in SCAD as compared with controls, this miRNA could also be a promising marker for atherosclerotic disease.

To substantiate that our findings are not merely due to chance, we wanted to show that at least one of these
miRNAs showed similar results as expected from the literature. Mir-122-5 $\mathrm{p}$ is known to be a liver-specific miRNA. Therefore, we expect that elevated miR-122-5 $\mathrm{p}$ is associated with increased liver enzymes. We observed a significant positive correlation with increasing levels of miR-122-5p with gamma-glutamyltransferase (gamma-GT) $\beta 0.08$ (95\% CI 0.06 to $0.09 ; \mathrm{p}<0.001)$ and ALAT $\beta 0.05$ (95\% CI 0.03 to $0.06 ; \mathrm{p}<0.001$ ). Besides, we also observed that gamma-GT and ALAT were significantly elevated in SCAD (mean \pm SD; for gamma-GT $35.5 \pm 1.9 \mathrm{U} / \mathrm{L}$; ALAT $30.5 \pm 1.6 \mathrm{U} / \mathrm{L}$ ) as compared with SubA (gamma-GT 27.7 $\pm 1.7 \mathrm{U} / \mathrm{L}$; ALAT $25.1 \pm 1.5 \mathrm{U} / \mathrm{L}$, $\mathrm{p}<0.05$ ) and controls (gamma-GT 23.0 $\pm 1.8 \mathrm{U} / \mathrm{L}$; ALAT $22.8 \pm 1.5 \mathrm{U} / \mathrm{L}, \mathrm{p}<0.05)$. Concerning the patients with $\mathrm{UCAD}$, only gamma-GT $(34.9 \pm 1.6 \mathrm{U} / \mathrm{L})$ was significantly elevated as compared with SubA $(27.7 \pm 1.7 \mathrm{U} / \mathrm{L}, \mathrm{p}<0.05)$ and controls $(23.0 \pm 1.8 \mathrm{U} / \mathrm{L}, \mathrm{p}<0.05)$.

\section{DISCUSSION}

In this study, we were able to show that tissue-derived miR-223-3 $p$ and serum miR-122-5 $p$ are robust and promising biomarkers for unstable CAD disease, possibly reflecting plaque instability. Besides, miR-122-5 p could also reflect an adverse metabolic profile increases the risk for atherosclerosis. The miRNAs miR-146-3 $p$, miR-155-5 $\mathrm{p}$ and miR-145-5 p might also be able to identify unstable CAD, however, the data were less robust, especially miR-233-3 $p$ seems a promising biomarker for individuals at risk for an acute coronary syndrome. MiR-122-5 p could be used to identify individuals with an adverse metabolic profile, such as insulin resistance at risk for unstable CAD.

This study does not aim to unravel the biochemical process underlying these candidate miRNAs but merely tries to robustly show the epidemiological evidence that they are promising biomarkers. We show that miR-223-3p both in tissue and in serum as well as in multiple independent cohorts show similar results. Many investigators have shown the relation between plaque-derived miRNAs or serum miRNAs in relation to CAD, but most of this research fails when it comes to validation, because of small sample errors and change finding. We managed to improve our miRNA measurement and analytical techniques as $\operatorname{such}^{22}$ that we were able to robustly validate the findings of miR-223-3 $p$. We only show that miR-223-3p is related to the acute phase of $\mathrm{CAD}$, while others have shown that miR-223-3p could be released from vascular endothelial cells ${ }^{2324}$ and that it might play a role in endothelial cell dysfunction, atherosclerotic lesion initiation, progression and possibly plaque rupture. ${ }^{25}{ }^{26}$ Therefore, miR-223-3 $p$ and maybe also miR-122-5 p, might originate from plaque tissue and directly reflect damaged endothelial cells of the plaque, on the edge of rupture. On the other hand, miR-122-5 $\mathrm{p}$ was the only miRNA that was also upregulated in SCAD. Therefore, miR-122-5p might rather be a marker of the atherosclerotic process or even the unfavourable metabolic profile underlying 
atherosclerosis. In line with these results, Niculescu et al also found a significant upregulation of miR-122-5 $p$ in patients with stable and unstable angina compared with controls. ${ }^{27}$ Besides, we were able to confirm the relationship of miR-122-5 $\mathrm{p}$ as a highly conserved liver miRNA regulating lipid metabolism ${ }^{28} 29$ and has been associated with liver diseases such as metabolic syndrome and type 2 diabetes, major risk factors for atherosclerosis. Its elevation among both the patients with SCAD and UCAD might reflect underlying liver pathology such as hepatic steatosis, which is related to atherosclerosis, ${ }^{3031}$ but we did not investigate this. Whether miR-122-5 $\mathrm{p}$ has a direct or indirect effect on atherosclerosis is still unknown, since it has also been suggested that miR-122-5 $\mathrm{p}$ is released from both the liver and endothelial cells. ${ }^{32} 33$

\section{Strengths and limitations}

One might argue that our findings are contradictory to the claim the studies make, we selected our candidate literature miRNAs from. Namely, these studies claim to have investigated miRNAs related to SCAD instead of UCAD. On the other hand, the cohorts we selected our candidate literature miRNAs from, did not truly represent SCAD. In some of these cohorts, blood was drawn at the time that these patients had complaints of angina ${ }^{2734-36}$ and interestingly, in one of these studies, CK-MB and troponin levels were even higher in the group of SCAD as compared with the group with UCAD. ${ }^{34}$ On the other hand, one might argue that also in our study, these markers simply reflect acute ischaemia, since blood was taken in the acute phase of an acute coronary syndrome. For most of our candidate miRNAs, this might very well be true, since miR-223, ${ }^{3738}$ miR-133a-3p, ${ }^{20}$ miR-146-3p $p^{27} 39$ and miR-155-5 $\mathrm{p}^{39}$ are known to be related to myocardial damage as could be shown by an elevation in the ischaemia markers miR-499-5 p and hsTnI levels. On the other hand, miR-122-5 p was apart from its upregulation in patients with UCAD also upregulated in patients with SCAD, and could not simply reflect ischaemia, since the miRNA-499-5 p and hsTnI levels were low in these patients.

Another limitation of our study is that the discovery phase was a comparison between miRNAs of plaque tissue of patients with SCAD and UCAD and not compared with the healthy vessel wall of controls. This is not possible, but on the other hand, this unique set-up is able to identify markers of plaque instability in a background of atherosclerosis. Regarding miR-223-3 p, this was the only miRNA that could be validated from the discovery phase, and might, therefore, reflect a true marker of plaque instability.

Most miRNAs form the discovery phase and could not be validated in the validation cohorts. This could be because miRNAs concentration is much higher in tissue as compared with blood and that the level in the circulation, although this might be higher as compared with controls, could still be too low to measure it reliably. If the lack of validation was due to technical differences in the measuring method, none of the miRNAs could have been measurable in the validation cohorts, which was not the case.

Another limitation comes from the fact that we used different source populations. Therefore, samples might differ, since they were handled differently and since the age of the source populations were different. We do not think this has affected the outcomes substantially since the expression levels of miR-125-5p, miR-193-3p and miR-126-3 p were quite similar among all the four groups. Besides, miRNAs are known for their stability. Therefore, longer storage times or slightly different handling of the samples are unlikely to affect miRNA expression levels. Finally, our data handling protocol takes into account technical differences.

One of our major strengths is that miRNA data handling is extremely thorough and handles missing values and technical difficulties in a systematic way, leading to more robust results. ${ }^{40}$ For instance, all miRNAs were measured in triplicates and interplate variance was taken into account as an extra normalisation procedure by the second validation study. Besides, normalisation was performed taking into account three endogenous miRNAs as well as two technical normalisers. Furthermore, extra quality checks were carried out by a prespecified data handling pipeline, which has been shown to increase both accuracy and precision when analysing circulating miRNAs. ${ }^{40}$ We think that the robust methods and large sample sizes used in this study make this one of the most extensive circulating miRNA studies in the cardiovascular research field so far. Moreover, the differences in miRNA expression between the multiple validation phases underscore the small sample error issues that circulating miRNA research often encounters and underscores the robustness of the miRNAs that could indeed be validated. Also in our study, validation of previous findings was difficult. In contrast, we were able to confirm most of the literature picked candidate miRNAs and we showed a consistent upregulation of miR-223-3 $p$ from discovery to second validation, again underscoring the robustness of the results in this study.

Finally, we would like to emphasise that we did not include speculations on the mechanism since we believe many other studies are prone to small sample error and therefore, the quality and robustness of many experimental small miRNA studies is questionable. For true mechanistic investigations, animal experiments are the preferred method thereafter to be validated in sufficiently large enough human studies. Therefore, this study was planned as a clinical study investigating biomarkers for UCAD.

\section{CONCLUSIONS}

In conclusion, miR-223-3 $p$ was the only miRNA that could be validated in both the validation cohorts and seems a promising marker for UCAD. Furthermore, miR-122-5 $\mathrm{p}$ and miR-223-3 $\mathrm{p}$ might even be markers 
of plaque instability and miR-122-5 p could also reflect an adverse metabolic profile that increases the risk for atherosclerosis.

\section{Author affiliations}

${ }^{1}$ Department of Vascular Medicine, Amsterdam UMC, Location AMC, The University of Amsterdam, Amsterdam, The Netherlands

${ }^{2}$ Department of Clinical Epidemiology, Biostatistics and Bioinformatics, Amsterdam UMC, Location AMC, The University of Amsterdam, Amsterdam, The Netherlands ${ }^{3}$ Department of Cardiology, Amsterdam UMC, Location AMC, The University of Amsterdam, Amsterdam, The Netherlands

${ }^{4}$ Department of Pathology, Amsterdam UMC, Location AMC, The University of Amsterdam, Amsterdam, The Netherlands

${ }^{5}$ Department of Experimental Vascular Medicine, Amsterdam UMC, Location AMC, The University of Amsterdam, Amsterdam, The Netherlands

${ }^{6}$ Molecular and Cellular Hemostasis, Sanquin Research, Amsterdam, The Netherlands

${ }^{7}$ Department of Experimental Cardiology, Amsterdam Cardiovascular Sciences, Amsterdam, The Netherlands

Acknowledgements The authors would like to thank Joost Leenders, Roelien Meijering, Zhen Liu from ACS Biomarker BV, the Netherlands (http://www. acsbiomarker.com) and Suzanne Battjes, AMC medical student, for the execution of the qPCR miRNA measurements in the validation experiments.

Contributors SS, MWJdR, S-JP-S, JCMM, EEC: conception and design of research. MWJdR, MGMK, BMS: material collection. MWJdR, MGMK, BMS: performed experiments. SS, MWJdR, MGMK, S-JP-S: analysis and interpretation. SS, MWJdR, MGMK: prepared figures. SS, MWJdR, MGMK: drafted manuscript. MAMB, RDW, ACvdW, BMS, JCMM, EEC, S-JP-S: edited and revised manuscript.

Funding This study was funded by BDDA PoC grant (2012); validation of miRNA biomarkers for unstable plaque and The European Union: FP7 (BestAgeing, GA 306031).

\section{Competing interests None declared.}

Patient consent for publication Not required.

Ethics approval This study complies with the Declaration of Helsinki. The study protocol for both biobank used in the first (MASS study biobank: 2013_220) and the second source population (biobank premature atherosclerosis: 2014_095) was approved by the biobank ethical committee of the Academic Medical Center in Amsterdam and all individuals gave written informed consent.

Provenance and peer review Not commissioned; externally peer reviewed.

Data availability statement Data are available on reasonable request.

Open access This is an open access article distributed in accordance with the Creative Commons Attribution Non Commercial (CC BY-NC 4.0) license, which permits others to distribute, remix, adapt, build upon this work non-commercially, and license their derivative works on different terms, provided the original work is properly cited, appropriate credit is given, any changes made indicated, and the use is non-commercial. See: http://creativecommons.org/licenses/by-nc/4.0/.

\section{ORCID iDs}

Sandeep Singh http://orcid.org/0000-0001-9338-1209

Esther E Creemers http://orcid.org/0000-0002-9001-3854

\section{REFERENCES}

1 Roth GA, Johnson C, Abajobir A, et al. Global, regional, and national burden of cardiovascular diseases for 10 causes, 1990 to 2015. J Am Coll Cardiol 2017;70:1-25.

2 Goff DC, Lloyd-Jones DM, Bennett G, et al. 2013 ACC/AHA guideline on the assessment of cardiovascular risk: a report of the American College of Cardiology/American heart association Task force on practice guidelines. Circulation 2014;129:S49-73.

3 Piepoli MF, Hoes AW, Agewall S, et al. 2016 European Guidelines on cardiovascular disease prevention in clinical practice: The Sixth Joint Task Force of the European Society of Cardiology and Other Societies on Cardiovascular Disease Prevention in Clinical Practice (constituted by representatives of 10 societies and by invited experts)Developed with the special contribution of the European Association for Cardiovascular Prevention \& Rehabilitation (EACPR). Eur Heart J 2016;37:2315-81.
4 Crea F, Liuzzo G. Pathogenesis of acute coronary syndromes. J Am Coll Cardiol 2013;61:1-11.

5 Libby P. Mechanisms of acute coronary syndromes and their implications for therapy. N Engl J Med 2013;368:2004-13.

6 Bartel DP. MicroRNAs: genomics, biogenesis, mechanism, and function. Cell 2004:116:281-97.

7 Chistiakov DA, Bobryshev YV, Orekhov AN. Changes in transcriptome of macrophages in atherosclerosis. J Cell Mol Med 2015;19:1163-73.

8 Bildirici AE, Arslan S, Özbilüm Şahin N, et al. MicroRNA-221/222 expression in atherosclerotic coronary artery plaque versus internal mammarian artery and in peripheral blood samples. Biomarkers 2018;23:670-5.

9 Romaine SPR, Tomaszewski M, Condorelli G, et al. MicroRNAs in cardiovascular disease: an introduction for clinicians. Heart 2015;101:921-8.

$10 \mathrm{He}$ L, Hannon GJ. MicroRNAs: small RNAs with a big role in gene regulation. Nat Rev Genet 2004;5:522-31.

11 Kim JS, Pak K, Goh TS, et al. Prognostic value of microRNAs in coronary artery diseases: a meta-analysis. Yonsei Med $\mathrm{J}$ 2018;59:495-500.

12 Gupta SK, Bang C, Thum T. Circulating microRNAs as biomarkers and potential paracrine mediators of cardiovascular disease. Circ Cardiovasc Genet 2010;3:484-8.

13 Mitchell PS, Parkin RK, Kroh EM, et al. Circulating microRNAs as stable blood-based markers for cancer detection. Proc Natl Acad Sci U S A 2008;105:10513-8.

14 Parahuleva MS, Lipps C, Parviz B, et al. MicroRNA expression profile of human advanced coronary atherosclerotic plaques. Sci Rep 2018;8:7823.

15 Lu Y, Thavarajah T, Gu W, et al. Impact of miRNA in atherosclerosis. Arterioscler Thromb Vasc Biol 2018;38:e159-70.

16 Busch A, Eken SM, Maegdefessel L. Prospective and therapeutic screening value of non-coding RNA as biomarkers in cardiovascular disease. Ann Transl Med 2016;4:236.

17 Ahlin F, Arfvidsson J, Vargas KG, et al. MicroRNAs as circulating biomarkers in acute coronary syndromes: a review. Vascul Pharmacol 2016;81:15-21.

18 Schulte C, Zeller T. microRNA-based diagnostics and therapy in cardiovascular disease-Summing up the facts. Cardiovasc Diagn Ther 2015;5:17-36

19 Navickas R, Gal D, Laucevičius A, et al. Identifying circulating microRNAs as biomarkers of cardiovascular disease: a systematic review. Cardiovasc Res 2016;111:322-37.

20 Corsten MF, Dennert R, Jochems S, et al. Circulating MicroRNA208b and MicroRNA-499 reflect myocardial damage in cardiovascular disease. Circ Cardiovasc Genet 2010;3:499-506.

21 Ruijter JM, Ramakers C, Hoogaars WMH, et al. Amplification efficiency: linking baseline and bias in the analysis of quantitative PCR data. Nucleic Acids Res 2009;37:e45.

22 Kok MGM, de Ronde MWJ, Moerland PD, et al. Small sample sizes in high-throughput miRNA screens: a common pitfall for the identification of miRNA biomarkers. Biomol Detect Quantif 2018;15:1-5.

23 Tabet F, Vickers KC, Cuesta Torres LF, et al. HDL-transferred microRNA-223 regulates ICAM-1 expression in endothelial cells. Nat Commun 2014;5:3292

24 Pan Y, Liang H, Liu H, et al. Platelet-secreted microRNA-223 promotes endothelial cell apoptosis induced by advanced glycation end products via targeting the insulin-like growth factor 1 receptor. $J$ Immunol 2014;192:437-46.

25 Gimbrone MA, García-Cardeña G. Endothelial cell dysfunction and the pathobiology of atherosclerosis. Circ Res 2016;118:620-36.

26 Damani S, Bacconi A, Libiger O, et al. Characterization of circulating endothelial cells in acute myocardial infarction. Sci Transl Med 2012;4:126ra33.

27 Niculescu LS, Simionescu N, Sanda GM, et al. MiR-486 and miR-92a identified in circulating HDL discriminate between stable and vulnerable coronary artery disease patients. PLoS One 2015;10:e0140958

28 Norata GD, Sala F, Catapano AL, et al. MicroRNAs and lipoproteins: a connection beyond atherosclerosis? Atherosclerosis 2013;227:209-15.

29 Elmén J, Lindow M, Silahtaroglu A, et al. Antagonism of microRNA-122 in mice by systemically administered LNA-antimiR leads to up-regulation of a large set of predicted target mRNAs in the liver. Nucleic Acids Res 2008;36:1153-62.

30 Willeit $\mathrm{P}$, Skroblin P, Moschen AR, et al. Circulating microRNA-122 is associated with the risk of new-onset metabolic syndrome and type 2 diabetes. Diabetes 2017;66:347-57. 
31 Tsai W-C, Hsu S-D, Hsu C-S, et al. MicroRNA-122 plays a critical role in liver homeostasis and hepatocarcinogenesis. J Clin Invest 2012;122:2884-97.

32 Li X, Yang Y, Wang L, et al. Plasma miR-122 and miR-3149 potentially novel biomarkers for acute coronary syndrome. PLoS One 2015;10:e0125430.

33 Li X-D, Yang Y-J, Wang L-Y, et al. Elevated plasma miRNA-122, $-140-3 p,-720,-2861$, and -3149 during early period of acute coronary syndrome are derived from peripheral blood mononuclear cells. PLoS One 2017;12:e0184256.

34 D'Alessandra Y, Carena MC, Spazzafumo L, et al. Diagnostic potential of plasmatic microRNA signatures in stable and unstable angina. PLoS One 2013;8:e80345.

35 Dong J, Liang Y-Z, Zhang J, et al. Potential role of LipometabolismRelated microRNAs in peripheral blood mononuclear cells as biomarkers for coronary artery disease. J Atheroscler Thromb 2017;24:430-41.
36 Gao W, He H-W, Wang Z-M, et al. Plasma levels of lipometabolismrelated miR-122 and miR-370 are increased in patients with hyperlipidemia and associated with coronary artery disease. Lipids Health Dis 2012;11:55.

37 Li C, Fang Z, Jiang T, et al. Serum microRNAs profile from genomewide serves as a fingerprint for diagnosis of acute myocardial infarction and angina pectoris. BMC Med Genomics 2013;6:16.

38 Schulte C, Molz S, Appelbaum S, et al. miRNA-197 and miRNA-223 predict cardiovascular death in a cohort of patients with symptomatic coronary artery disease. PLoS One 2015;10:e0145930.

39 Zidar N, Boštjančič E, Glavač D, et al. MicroRNAs, innate immunity and ventricular rupture in human myocardial infarction. Dis Markers 2011;31:259-65.

40 de Ronde MWJ, Ruijter JM, Lanfear D, et al. Practical data handling pipeline improves performance of qPCR-based circulating miRNA measurements. RNA 2017;23:811-21. 